\title{
Reliability analysis of the existing reinforced concrete beams with normal cracks by rebar strength criteria
}

\author{
Sergey A. Solovyev ${ }^{1,{ }^{*}}$, Anastasia A. Solovyeva ${ }^{1}$, Alexander A. Kochkin ${ }^{1}$, Timur R. Akhmetov ${ }^{2}$ \\ ${ }^{1}$ Vologda State University, Industrial and Civil Construction Department, Russian Federation \\ ${ }^{2}$ Kazan State Power Engineering University, str. Krasnoselskaya, 51, 420066, Kazan, Russia
}

\begin{abstract}
The paper describes the problem of the reliability analysis for individual reinforced concrete (RC) beams by the rebar strength criterion in cross section with a normal crack. It is proposed to evaluate the stress in the rebar by measurements of the crack width at the level of the rebar using the functional dependence of the crack width and the strain (deformation) in the reinforcement. It is also proposed to replace the modulus of elasticity of steel reinforcement on the secant modulus of elasticity, taking into account the increased reinforcement strain in cross section with crack. The work considers two options of the crack width in beam: less and greater than the ultimate crack width. The reliability analysis of RC beams by the rebar strength is based on the possibility theory and fuzzy set theory by the reason of small statistical data from measurements on existing individual RC beams. The use of offered reliability analysis methods will allow preventing the failures of reinforced concrete beams and in some cases to obtain economic benefit from the possibility of further operation of RC beams with cracks, even with a crack width more than ultimate value.
\end{abstract}

\section{Introduction}

Mechanical safety is the state of buildings and structures in which there is no unacceptable risk of a structural failure. According to the Eurocode 0 "Basis of structural design", the reliability is an ability of a structure or a structural part to meet the specified requirements, including the design working life, for which it has been designed. Reliability is usually expressed in probabilistic terms. General principles of structural reliability are regulated by Eurocode 0 and International Standard "General principles on reliability of structures". The Interstate Standard GOST 27751-2014 "Reliability of structures and foundation soils" regulates the general principles of structural reliability in the Russian Federation.

The failures of structures, including reinforced concrete beams and slabs, testify to the insufficient implementation of methods for the reliability analysis of structures during their inspection. It also shows the lack of methods and techniques for reliability (safety) analysis of structures on some limit state criteria. The measure of structural element reliability is probability of non-failure by all limit state criteria. The limit state criteria for reinforced concrete beams are: rebar and concrete strength, deflection, cracking, crack widths, etc. This article will consider the method for reliability analysis of existing RC beams with normal cracks on rebar strength.

Microcracks naturally present in the concrete of the existing reinforced concrete structures subject to the influence of various factors. The microcracks can become macrocracks. A series of normal cracks in beams are often formed in the tensile zone of the beam under the influence of a distributed load [1]. The ultimate crack width $a_{c r c, u l t}$ is regulated by Standards and Codes (Eurocode 2, ACI 318-19, The Russian Design requirements SP 63.13330.2018, etc.) depending on the purpose of structure, the environment, type and diameter of the rebar etc. The stress in the rebar $\sigma_{s}$ and in the compressed zone of concrete $\sigma_{b}$ increase dramatically in cross-section with a crack of the reinforced concrete beam. Fig. 1 contains fragments of the beam with cracks and the diagram of stress in the rebar and concrete with the local increase of stress.

* Corresponding author: solovevsa@ vogu35.ru 


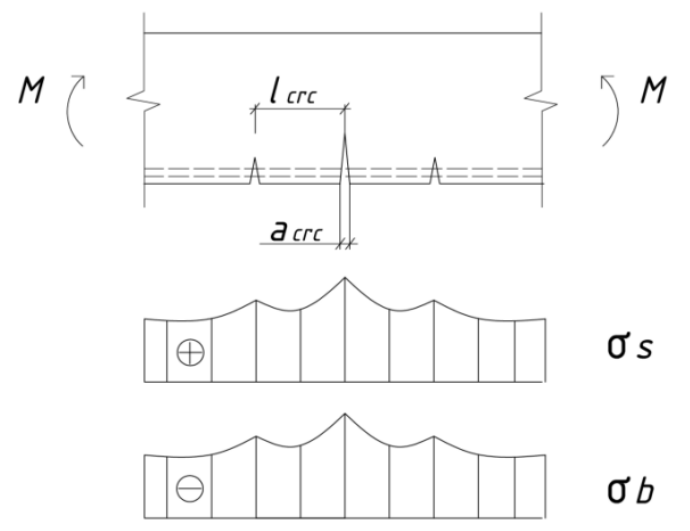

Fig. 1. Fragment of the RC beam with cracks and stress diagrams $\sigma_{s}$ and $\sigma_{b}$.

The cracking leads to a decrease of load-bearing capacity and reliability (safety) of reinforced concrete beams, even in case when $a_{c r c} \leq a_{c r c, u l t}$.

The condition $a_{c r c} \leq a_{c r c, u l t}$ doesn't mean that the safety of the existing reinforced concrete beam is provided by the rebar strength. And the condition $a_{c r c}>a_{c r c, u l t}$ does not mean that the reinforced concrete beam must be dismantled by the criterion of rebar strength. For example, Soviet Departmental Building Code VSN (R) 53-86 "Rules for the evaluation of residential buildings deterioration" recommends only injection into the crack of cement and coating the beams with cement mortar with a width of cracks in reinforced concrete beams up to $1 \mathrm{~mm}$. The area of beams with the crack is also covered by epoxy resin. The same applies to reinforced concrete ladders and other structural elements.

The paper [1] describes the evaluation of ultimate load by values of the mean and standard deviation of random variable (crack width $\tilde{a}_{c r c}$ ); ultimate crack width $a_{c r c, u l t}$ and stress in rebar $\sigma_{s}$ is constant values. This method uses the linearization method and probability of failure as $P=\Phi\left(-\beta_{0}\right)$, where $\Phi\left(-\beta_{0}\right)$ is the Gauss error function, where $\beta_{0}=m / s$, where $m$ is a mean value; $s$ is a standard deviation. However, the distance between the cracks $l_{S}$ is not taken into account in [1]. Moreover, it is difficult to get the full statistical data for individual reinforced concrete beam because of large variability of some statistical parameters.

The work [2] considers experimental and theoretical calculation of the reliability index for reinforced concrete beams with different cross-sections. The paper [3] describes the calculation of the non-failure probability (reliability index) of reinforced concrete beams with carbon-fiber reinforced polymers (CFRP). A statistical analysis to control cracking in reinforced concrete structures based on the "68-95-99.7 rule" is presented in [4]. The paper [5] discusses the risk assessment of corrosion in the rebar of concrete structures and evaluation of their reliability and durability by probabilistic and statistical methods. The paper [6] discusses the measurement and monitoring of the crack width using piezo-ceramic sensors. The work [7] presents a study of the durability of reinforced concrete beams with cracks under the influence of corrosive environments and the influence of the crack width on corrosion of rebar. The reliability analysis of reinforced concrete beams using different probabilistic and statistical methods are also discussed in [8-11].

The considered literature shows the possibility of crack width measuring in reinforced concrete beams, and its influence on the load-bearing capacity and reliability (safety) of reinforced concrete elements. However, to the best of our knowledge, methods of reliability analysis according to the criterion of the rebar strength in the cross sections with crack are not thoroughly investigated in the scientific literature. In particular, they are not applied to reinforced concrete beams with various statistical information about controlled parameters in mathematical models of limit state. So, this consideration is the theme of the present work.

\section{Methods}

The mathematical model of the limit state for reliability analysis of $\mathrm{RC}$ beam on rebar strength can be written as:

$$
\tilde{\sigma}_{s} \leq \tilde{\sigma}_{s, u l t}
$$

where $\tilde{\sigma}_{s}$ is the stress in a rebar in the beam crosssection with a crack; it is a random variable (marked by a wavy line above the symbol); $\tilde{\sigma}_{s, u l t}$ is the ultimate tensile stress of rebar, which was evaluated experimentally from the rebar samples tensile tests. It is proposed to use the yield strength $\sigma_{0.2}$ or the elastic limit $\sigma_{0.02}$ as the $\tilde{\sigma}_{S, u l t}$ value.

According to the Set of Rules SP 63.13330.2018 "Concrete and reinforced concrete structures", the crack width is calculated as

$$
a_{c r c}=\sigma_{s} \varphi_{1} \varphi_{2} \varphi_{3} \psi_{s} l_{s} / E_{s}
$$

where $l_{s}$ is the distance between the normal cracks in the tensile zone of reinforced concrete beams; $E_{S}$ is the modulus of elasticity of steel rebars; $\varphi_{1}$ is the factor of the load action (1.0 for a short load action; 1.4 for a long load action); $\varphi_{2}$ is the factor of the profile of the longitudinal reinforcement: $\varphi_{2}=0.5$ for the ribbed bars; $\varphi_{2}=0.8$ for the plain bars; $\varphi_{3}$ is the factor of a structural element behavior: $\varphi_{3}=1$ for flexural elements and $\varphi_{3}=1.2$ for tensile elements; $\psi_{s}$ is the Murashev coefficient: $\psi_{s}=1$ if $a_{c r c} \leq a_{c r c, u l t}$ and $\psi_{s}<1$ if $a_{\text {crc }}>a_{\text {crc }, \text { ult }}$.

It is proposed to use this equation to determine the rebar stress $\sigma_{S}$ in the beam cross-section with a crack by the measurements of the crack width. In this case, $\sigma_{S}$ is determined as: 


$$
\tilde{\sigma}_{s}=\tilde{a}_{c r c} E_{s} / \varphi_{1} \varphi_{2} \varphi_{3} \tilde{\psi}_{s} \tilde{l}_{s}
$$

The Murashev coefficient is evaluated as $\psi_{s}=1-0.8 \frac{M_{c r c}}{M}$, where $M_{c r c}=R_{b t, s e r} W_{p l}$ or $M_{c r c}=\sigma_{b t} W_{p l} ; R_{b t, s e r}$ is a tensile strength of concrete; $\sigma_{b t}$ is an ultimate tensile stress in concrete; $W_{p l}$ is an elastic-plastic moment of resistance.

Taking into account eq. (2) and condition $a_{c r c} \leq a_{c r c, u l t},(1)$ can be represented as:

$$
\tilde{a}_{c r c} E_{s} / \varphi_{1} \varphi_{2} \varphi_{3} \tilde{l}_{s} \leq \tilde{\sigma}_{s, u l t}
$$

and if $a_{c r c}>a_{c r c, u l t}$ :

$$
\tilde{a}_{c r c} E_{s} / \varphi_{1} \varphi_{2} \varphi_{3} \tilde{\psi}_{s} \tilde{l}_{s} \leq \tilde{\sigma}_{s, u l t}
$$

In [12] academician I. N. Karpenko proposed to correct the equation (3'). Here $E_{S}$ is replaced by the secant modulus of elasticity $E_{S}^{\mathrm{sec}}$, which is less than the standard value of $E_{S}=2 \cdot 10^{11} \mathrm{~Pa}$. The $E_{S}^{\mathrm{sec}}$ is determined (in the inspection and reliability analysis of reinforced concrete beams) by the tensile tests of rebar samples from reinforcement beams and by the stressstrain $\sigma-\varepsilon$ diagrams. It is proposed to calculate $\sigma^{\prime}{ }_{S}$ by

(2) as $\sigma_{s}{ }^{\prime}=a_{c r c} E_{s}^{\mathrm{sec}} / \varphi_{1} \varphi_{2} \varphi_{3} \psi_{s} l_{s}$ by the results of the $a_{c r c}$ and $l_{s}$ measurements.

Consider the option of reliability analysis of individual reinforced concrete beam according to the criterion of the rebar strength (1) in cross-section with a maximum crack width as $a_{c r c} \leq a_{c r c, u l t}$ and $\psi_{s}=1$. We introduce the notations $\tilde{a}_{c r c}=X, \tilde{l}_{s}=Y$, $\tilde{\sigma}_{s, u l t}=Z, \psi_{s}=1, \varphi_{1} \varphi_{2} \varphi_{3} / E_{S}^{\mathrm{sec}}=k$. Then:

$$
X / Y \cdot Z \leq k
$$

where $k$ is a constant value; $X, Y, Z$ are fuzzy variables by the reason of small statistical data in the individual reinforced concrete beam.

\section{Results}

Consider the reliability analysis of reinforced concrete beam according to the mathematical model (4) by the theory of possibility approach [13] using the Zadeh's principle from the fuzzy sets theory [14]. The possibility distribution function $\pi_{X}(x)$ can be accepted as:

$$
\pi_{X}(x)=\exp \left[-\left(\frac{x-a_{x}}{b_{x}}\right)^{2}\right]
$$

where

$$
a_{x}=0.5 \cdot\left(X_{\max }+X_{\min }\right) ;
$$

$b_{x}=\left(X_{\max }-X_{\min }\right) / \sqrt{-\ln \alpha}, X_{\max }$ and $X_{\min }$ are the maximum and minimum values in the subset $\{x\}$ of fuzzy variable $X$ from the test results; $\alpha \in[0 ; 1]$ is a cut (risk) level. The reverse function of $\pi_{X}(x)$ from (5) is $x=a_{x} \pm b_{x} \sqrt{-\ln \alpha_{*}} \quad$ or $\quad x=a_{x} \pm b_{x} \beta$, where $\beta=\sqrt{-\ln \alpha_{*}}$.

Further, the fuzzy function $G$ can be created from fuzzy arguments $X, Y, Z$ in accordance with the Zadeh's principle as:

$$
G=X / Y \cdot Z \leq k
$$

Fuzzy function $G$ is characterized by the distribution function $\pi_{G}(g)$ with the average value $a_{g}=a_{x} / a_{y} a_{z} a_{t}$ and with the left $g \leq \alpha_{g}$ and the right $g>\alpha_{g}$ branches. The reverse function $g$ of $G$ will be determined through the reverse functions $x, y, z$ from $X$, $Y, Z$ as described above.

From the left branch of $\pi_{G}(g)$, we have:

$$
g_{\text {left }}=\left(a_{x}-b_{x} \beta\right) /\left(a_{y}+b_{y} \beta\right)\left(a_{z}+b_{z} \beta\right),
$$

and for the right branch:

$$
g_{\text {right }}=\left(a_{x}+b_{x} \beta\right) /\left(a_{y}-b_{y} \beta\right)\left(a_{z}-b_{z} \beta\right),
$$

where $\beta=\sqrt{-\ln \pi_{G}(g)}=\sqrt{-\ln \alpha_{*}}$.

A minus sign is placed before the " $b$ " term if from this value the $g_{\text {left }}$ value increases. We have $\pi_{G}(g)=1$ or $\beta=0$ in case $g=a_{g}=a_{x} / a_{y} a_{z} a_{t}$. The value of nonfailure possibility $R$ is taken $R=1$ if $a_{g} \leq k$ in (7'). The value of failure possibility $Q$ (for the right branch of $\left.\pi_{G}(g)\right)$ is calculated by $\beta$ from (7') with $g_{u l t}=k$, which corresponds to the lowest reliability. By the results of solving ( $\left.7^{\prime}\right), \beta_{\min }$ is determined by the absolute value with $g_{u l t}=k$, and the possibility of failure on the rebar strength criterion is $Q=\exp \left(-\beta_{\min }^{2}\right)$

A fuzzy variable is characterized by the possibility $R$ and the necessity $N$. The necessity $N$ of the beam nonfailure is calculated as $N=1-Q$. The reliability of reinforced concrete beam by the rebar strength criterion in cross-section with a crack is characterized by interval $[N ; R=1]$ or by $[\underline{P} ; \bar{P}]$ in probabilistic notations, where $\underline{P}$ and $\bar{P}$ are upper and lower values of non-failure probability (reliability).

Algorithms for reliability analysis based on the proposed approaches can be considered using numerical examples. The initial data for the examples can be obtained as a result of inspections of reinforced concrete beams and of testing the control samples of steel reinforcement and concrete. A detailed description of the 
tests procedures is given in the relevant regulatory documents and standards.

Example 1. Consider the example of the reliability analysis for a reinforced concrete beam with the following data: $E_{S}^{\mathrm{sec}}=2 \cdot 10^{10} \mathrm{~Pa} ; \varphi_{1}=1.4, \varphi_{2}=0.5$, $\varphi_{3}=1$ are set by the SP 63.13330.2018. $\tilde{a}_{c r c}=\{0.24 ; 0.26 ; 0.28\} \quad \mathrm{mm} ; \quad \tilde{l}_{s}=\{0.17 ; 0.14 ; 0.11\} \quad \mathrm{m}$. The ultimate crack width $a_{c r c, u l t}$ is $0.30 \mathrm{~mm}$ as adopted in Table 4.1 of ACI 224R-01 for humidity, moist air or soil exposure conditions. By three tension tests of reinforcement bars control samples, the following data is obtained: $\tilde{\sigma}_{s, u l t}=\{240 ; 265 ; 215\} \mathrm{MPa}$.

The results of calculation using equation (5) are: $a_{x}=0.26 \mathrm{~mm} ; a_{y}=0.14 \mathrm{~m} ; a_{z}=240 \mathrm{MPa}$, $b_{x}=0.023 \mathrm{~mm} ; b_{y}=0.035 \mathrm{~m} ; b_{z}=28.9 \mathrm{MPa}$ with cut (risk) level $\alpha=0.05 ; \quad k=\varphi_{1} \varphi_{2} \varphi_{3} / E_{S}^{\mathrm{sec}}=3.5 \cdot 10^{-11}$ $\mathrm{m}^{2} / \mathrm{N}$.

$a_{g}=a_{x} / a_{y} a_{z} a_{t}=0.77 \cdot 10^{-11} \leq k=3.50 \cdot 10^{-11}$

$\mathrm{m}^{2} / \mathrm{N}$, then possibility of non-failure is $R=1$. From (7') for right branch $\pi_{G}(g)$ with $g_{\text {right }}=k$ as the most prudent solution: $\beta=\{2.46 ; 10.48\}$ and $\beta_{\min }=2.46$. The possibility of failure is $Q=\exp \left[-(2.46)^{2}\right\rfloor=0.0024$. The necessity $N$ of non-failure is $N=1-0.0024=0.9976$.

Reliability of reinforced concrete beam according to the rebar strength criterion is characterized by the interval $[0.9976 ; 1]$.

This example shows that even when the crack width is less than the ultimate crack width, the probability of failure of a reinforced concrete beam by the rebar strength criterion $\underline{P}=0.9976$ may be unacceptable, if the ultimate reliability should be more 0.998. For example, in Eurocode 0 "Basis of structural design" it is stated that reliability index should not be below 3.8 or probabilistic indicators 0.9999 during 50 years of operation for the structures of RC2 class.

Consider the option with $a_{c r c}>a_{c r c, u l t}$, but not more than $0.5 \mathrm{~mm}$. The Murashev coefficient $\psi_{s}$ is calculated as $\psi_{s}=1-0.8 \frac{M_{c r c}}{M}$. If $\psi_{s}$ is taken as a constant value, then reliability analysis will not differ from the above-considered algorithm. Further we consider a more complex version with a parameter $\psi_{s}$ as a random variable, which was determined by measurements of $M_{c r c}$. Let $\tilde{a}_{c r c}=X, \tilde{l}_{S}=Y$, $\tilde{\sigma}_{n p, s}=Z, \tilde{\psi}_{s}=T, \varphi_{1} \varphi_{2} \varphi_{3} / E_{S}^{\mathrm{sec}}=k$, then:

$$
X / Y \cdot Z \cdot T \leq k
$$

where $k$ is a constant value; $X, Y, Z, T$ are fuzzy variables by the reason of small statistical data in the individual reinforced concrete beam.

The fuzzy variables in (8) are described by the distribution function of possibilities (5). In accordance with the Zadeh's principle, the fuzzy function $J$ of fuzzy arguments $X, Y, Z, T$ from (8) can be represented as $J=X / Y \cdot Z \cdot T \leq k$. The fuzzy function $J$ is characterized by a distribution function $\pi_{J}(j)$ with left $j \leq \alpha_{j}$ and right $j>\alpha_{j}$ branches. The reverse function of $j$ from $J$ is determined from reverse functions of fuzzy variables $X, Y, Z, T$, which take the form of the reverse function of $x$ from $X$ as shown above for $X$. For the left and right branches $\pi_{J}(j)$ :

$$
\begin{aligned}
& j_{\text {left }}=\left(a_{x}-b_{x} \beta\right) /\left(a_{y}+b_{y} \beta\right)\left(a_{t}+b_{t} \beta\right)\left(a_{z}+b_{z} \beta\right),(9) \\
& j_{\text {right }}=\left(a_{x}+b_{x} \beta\right) /\left(a_{y}-b_{y} \beta\right)\left(a_{t}-b_{t} \beta\right)\left(a_{z}-b_{z} \beta\right),(9)
\end{aligned}
$$

where $\beta=\sqrt{-\ln \pi_{J}(j)}$. The function $\pi_{J}(j)=1$ or $\beta$ $=0$ in case of $a_{j}=a_{x} / a_{y} a_{z} a_{t}$. The value of nonfailure possibility $R$ is taken $R=1$ if $a_{j} \leq k$ in (9'). The failure possibility $Q$ (for the right branch of $\pi_{J}(j)$ ) is calculated by $\beta$ from (9') with $j_{u l t}=k$, which corresponds to the lowest reliability. By the results of solving ( $\left.9^{\prime}\right), \beta_{\min }$ is determined by the absolute value with $j_{u l t}=k$, and the possibility of failure on the rebar strength criterion is evaluated as $Q=\exp \left(-\beta_{\min }^{2}\right)$. The necessity $N$ of the beam non-failure is $N=1-Q$. The reliability of reinforced concrete beam by the rebar strength criterion in cross-section with a crack is characterized by interval $[N ; R=1]$ or by $[\underline{P} ; \bar{P}]$ in the probabilistic notations.

Example 2. Given: $a_{c r c, u l t}=0.30 \quad \mathrm{~mm}$; $E_{S}^{\mathrm{sec}}=2 \cdot 10^{10} \quad \mathrm{~Pa} ; \quad \varphi_{1}=1.4 ; \quad \varphi_{2}=0.5 ; \quad \varphi_{3}=1 ;$ $\tilde{a}_{c r c}=\{0.38 ; 0.36 ; 0.37\} \mathrm{mm} ; \tilde{l}_{s}=\{0.15 ; 0.13 ; 0.14\} \mathrm{m}$; $\widetilde{\sigma}_{0.2}=\{240 ; 215 ; 265\} \quad \mathrm{MPa}, \quad \tilde{\psi}_{s}=\{0.69 ; 0.71 ; 0.70\}$. So $a_{x}=0.37 \mathrm{~mm} ; a_{y}=0.14 \mathrm{~m} ; a_{z}=240 \mathrm{MPa}$, $b_{x}=0.012 \mathrm{~mm} ; b_{y}=0.012 \mathrm{~m} ; b_{z}=28.9 \mathrm{MPa}$ with cut (risk) level of $\alpha=0.05$; then $k=\varphi_{1} \varphi_{2} \varphi_{3} / E_{s}^{\mathrm{sec}}=3.5 \cdot 10^{-11} \quad \mathrm{~m}^{2} / \mathrm{N}$. As $a_{j}=a_{x} / a_{y} a_{z} a_{t}=1.57 \cdot 10^{-11} \leq k=3,5 \cdot 10^{-11} \quad \mathrm{~m}^{2} / \mathrm{N}$, then possibility of non-failure is $R=1$. From (10) for right branch $\pi_{J}(j)$ with $j_{u l t}=k$ as the most prudent solution: $\beta_{\min }=2.74$. The possibility of failure is $Q=\exp \left[-(2.74)^{2}\right]=0.0004$. The necessity $N$ of nonfailure is $N=1-0.0004=0.9996$. Reliability of reinforced concrete beam according to the rebar strength criterion is characterized by the interval $[0.9996 ; 1]$.

The example shows that even when the crack width is more ultimate value, which equal to $0.3 \mathrm{~mm}$, the probability of failure can be higher than the ultimate value $P_{u l t}=0.9995$ for $\mathrm{RC} 1$ structures according to Eurocode 0 . It suggests, that by one criterion - the crack 
width $a_{c r c}$ and it ultimate value $a_{c r c, u l t}$, it is impossible to evaluate the structural safety.

\section{Discussion}

In addition, the reliability of a reinforced concrete beam as the mechanical system should be estimated by the theorem of probabilities multiplication as $P=\prod_{i}^{n} P_{i}$, where $P_{i}$ is the probability of non-failure by $i$-criterion of limit state. This paper presents the method of reliability analysis by only one of the limit state criteria (1).

Consider the case of beam reliability analysis, in which statistical information about the parameter $\tilde{l}_{s}$ allows to set the probability distribution function for $\tilde{l}_{s}$, for example in the form of a normal (Gaussian) distribution with probability density function (PDF):

$$
f_{Y}(y)=\frac{1}{\sqrt{2 \pi} S_{y}} \exp \left[-\frac{\left(y-m_{y}\right)^{2}}{2 S_{y}^{2}}\right]
$$

where $m_{y}$ is the mean and $S_{y}$ is the standard deviation of random variable $Y=\tilde{l}_{s}$, which is calculated from the measurements of $\tilde{l}_{s}$. Consider the option with $a_{c r c} \leq a_{c r c, u l t}$. In this case $\psi_{s}=1$. The other parameters in (4) are fuzzy variables. Represent (4) with $\psi_{s}=1$ as:

$$
X / Z \leq Y \cdot k
$$

The fuzzy variable $T=X / Z$ and its values (the arguments) $t$ can be introduced. For the function $\pi_{T}(t)$ on the abscissa-axis $t \leq a_{x} / a_{z}$ (left branch) with the description of $X$ and $Z$ by distribution functions (5), the distribution function of possibilities $\pi_{T}(t)$ can be presented as:

$$
\pi_{T}(t)=\exp \left[-\left(\frac{a_{x}-a_{z} t}{b_{x}+b_{z} t}\right)^{2}\right]
$$

The same expression $\pi_{T}(t)$ will be for $t>a_{x} / a_{z}$ (the right branch). A probability $P_{T}(t)$ of condition $Y k \geq t$ is used to analyse the reliability of the reinforced concrete beam by (11). This probability $P_{T}(t)$ is generally calculated by the known methods of the probability theory from the expression $P_{T}(t)=\int_{s}^{\infty} f_{Y k}(y) d y=F_{Y}(t)$, where $\quad F_{Y}(t)$ is the distribution function of $Y$ at the point $t$. The cut (risk) level is set as $\alpha * \in[0 ; 1]$. The upper value of the probability is $\bar{P}(t)=\Phi\left(\frac{m_{Y} k-t^{*}}{s_{y} k}\right)$, where $t^{*}$ is determined from the equation $\pi_{T}\left(t^{*}\right)=\exp \left[-\left(\frac{a_{x}-a_{z} t^{*}}{b_{x}+b_{z} t^{*}}\right)^{2}\right]$ with $\pi_{T}\left(t^{*}\right)=\alpha *$ in form $t^{*}=\frac{a_{x}-b_{x} \sqrt{-\ln \alpha *}}{a_{z}+b_{z} \sqrt{-\ln \alpha *}}$. Similarly, the lower value of the probability can be evaluated as $\underline{P}(t)=\Phi\left(\frac{m_{Y} k-t * *}{S_{y} k}\right)$, where $t * *$ is determined from the right branch $\pi_{T}(t)$ and is equal to $t * *=\frac{a_{x}+b_{x} \sqrt{-\ln \alpha *}}{a_{z}-b_{z} \sqrt{-\ln \alpha *}}$.

Example 3. Here we use the data from the example 1: $t^{*}=7.59 \cdot 10^{-13} \mathrm{~m} / \mathrm{Pa}$ and $t^{* *}=15.78 \cdot 10^{-13} \mathrm{~m} / \mathrm{Pa}$. Assume $m_{y}=0.14 \mathrm{~m} ; S_{y}=0.025 \mathrm{~m}$. Then:

$$
\begin{aligned}
& \underline{P}(t)=\Phi\left(\frac{m_{Y} k-t * *}{S_{y} k}\right)= \\
& =\Phi\left(\frac{0.14 \cdot 3.5 \cdot 10^{-12}-15.78 \cdot 10^{-13}}{0.03 \cdot 3.5 \cdot 10^{-12}}\right)= \\
& =\Phi(3.18)=0.9985 \\
& \bar{P}(t)=\Phi\left(\frac{m_{Y} k-t^{*}}{S_{y} k}\right)=\Phi(3.94)=0.9999
\end{aligned}
$$

The reliability of reinforced concrete beam is characterized by interval $[0.9985 ; 0.9999]$, that is more informative than the interval $[0.9976 ; 1]$ in example 1 .

Consider the option with $a_{c r c}>a_{c r c, u l t}$, but not more than $0.5 \mathrm{~mm}$. Assume $\tilde{l}_{s}=Y$, the random variable with normal distribution (10). The parameter $\tilde{\psi}_{S}=T$ is adopted as fuzzy variable as described above in example 2. Then, by analogy with the performed reliability analysis for the case $a_{c r c} \leq a_{c r c, u l t}$,

$$
\begin{aligned}
& t^{*}=\frac{a_{x}-b_{x} \sqrt{-\ln \alpha *}}{\left(a_{z}+b_{z} \sqrt{-\ln \alpha *}\right)\left(a_{t}+b_{t} \sqrt{-\ln \alpha_{*}}\right)} \quad \text { and } \\
& \left.t^{* *}=\frac{a_{x}+b_{x} \sqrt{-\ln \alpha *}}{\left(a_{z}-b_{z} \sqrt{-\ln \alpha_{*}}\right)\left(a_{t}-b_{t} \sqrt{-\ln \alpha_{*}}\right.}\right) \cdot \text { Upper and }
\end{aligned}
$$
lower values of non-failure probability are determined 


$$
\begin{aligned}
& \text { by the equations } \bar{P}(t)=\Phi\left(\frac{m_{Y} k-t^{*}}{S_{y} k}\right) \quad \text { and } \\
& \underline{P}(t)=\Phi\left(\frac{m_{Y} k-t^{* *}}{S_{y} k}\right) \text {, respectively. }
\end{aligned}
$$

Example 4. Here we use the data from the example 2: $t^{*}=1.58 \cdot 10^{-12} \mathrm{~m} / \mathrm{Pa}$ and $t^{* *}=3.18 \cdot 10^{-12} \mathrm{~m} / \mathrm{Pa}$. Assume $\quad m_{y}=0.14 \mathrm{~m} ; S_{y}=0.01 \mathrm{~m}$. Then $\underline{P}(t)=\Phi\left(\frac{m_{Y} k-t^{* *}}{S_{y} k}\right)=\Phi\left(\frac{0.14 \cdot 3.5 \cdot 10^{-11}-3.18 \cdot 10^{-12}}{0.01 \cdot 3.5 \cdot 10^{-11}}\right)=0.9999$ . $\bar{P}(t)=1$. The reliability of reinforced concrete beam is characterized by interval $[0.9999 ; 1]$, that is more informative than the interval $[0.9996 ; 1]$ in the example 2.

Thus, precision of results of reliability analysis is increased with the growing of statistical data on the parameter $\tilde{l}_{S}=Y$.

The examples 1-4 show that normal cracks in reinforced concrete beams can be dangerous even if the width is less than $0.3 \mathrm{~mm}$ or the other ultimate value by any standard. Conversely, it is possible to allow the operation of reinforced concrete beams with normal cracks greater than the ultimate values, because their reliability index by the criterion of reinforcement bars strength can be quite high. In this case, special measures should be taken to protect the reinforcement bars from corrosion.

\section{Conclusions}

1. The article proposes new approaches of structural reliability analysis for the existing reinforced concrete beams by the rebar strength criterion in cross-section with a crack. The proposed approaches take into account different statistical data on the controlled parameters in the design mathematical models of a limit state;

2 . The choice of reliability analysis method depends on the amount of statistical data on random variables. The larger is the volume of statistical information, the narrower (more informative) the reliability interval is. If the reliability interval is too wide for decision-making or risk analysis, additional tests of control samples should be performed. The most accurate reliability value can be obtained by identifying the exact probability distribution function and its parameters for each random variable in the mathematical model of limit states;

3. The use of the described reliability analysis methods allow one to prevent the failures of reinforced concrete beams with normal cracks, and to obtain economic benefit from the possibility of further operation of reinforced concrete beams with normal cracks in some cases.

\section{References}

1. B.S. Rastorguev., V.S. Dahi. Limit-state calculation of reinforced concrete structures with the use of probabilistic method. Ind. and Civ. Eng., 7 (2016).

2. M. Al-Ansari. Reliability and flexural behavior of triangular and T-reinforced concrete beams. Int. jour. of Adv. Struct. Eng., 7 (2015).

3. H.D. Mohammad, J. Fadaee. Probabilistic assessment of torsion in concrete beams externally strengthened with CFRP composites. Mat. and Struct., 47 (2014).

4. E.J. Eom, W.S. Jang, K. Sang-chul. Managing concrete crack information through correction of the slab rebar arrangement based on Six Sigma. KSCE Jour. of Civ Eng., 19 (2015).

5. C. Chien-Kuo, F.C. Tu, C.Y. Fan. Risk assessment of environmental corrosion for reinforcing steel bars embedded in concrete in Taiwan. Natu. Haz., 75 (2015).

6. D. Hughi, H. Marzouk. Crack width monitoring system for reinforced concrete beams using piezoceramic sensors. Jour. of Civ.Struc. Heal. Monit., 5 (2015).

7. J. Torres, C. Andrade. Influence of Crack Width on Long Term Degradation of Concrete Structures. Durab. of Reinf. Conc. from Comp. to Prot. 2013.

8. A.F. Sharvil, N. Nikil, G. Shiddhartha. Reliability of a corroded RC beam based on Bayesian updating of the corrosion model. Eng. Struct., 126 (2016).

9. M. Shahnewaz, R. Machial, A. Shahria A. Optimized shear design equation for slender concrete beams reinforced with FRP bars and stirrups using Genetic Algorithm and reliability analysis. Eng. Struct., 107 (2016).

10. H. Xiea, Y. Wanga., R. Zoub. Reliability analysis of $\mathrm{RC}$ T-beam highway bridges in China based on a virtual bridge dataset. Eng. Struct., 104 (2015).

11. D. Bigaud, O. Ali. Time-variant flexural reliability of RC beams with externally bonded CFRP under combined fatigue-corrosion actions. Reliability Engineering \& System Safety, 131 (2014).

12. N.I. Karpenko, B.S. Sokolov, O.V. Radaykin. To assess the strength, the stiffness, the formation of cracks and their disclosure in the zone of pure bending of the reinforced concrete beams with the application of a nonlinear deformation model. News of High. Educ. Inst. Constr., 7 (2016).

13. D. Dubois, H. Prade. Possibility Theory, Probability Theory and Multiple-valued Logics: A Clarification. Ann. of Math. and Arti. Intell., 32 (2001).

14. L.A. Zadeh. Fuzzy sets. Inf. and Cont.,3 (1965). 